\title{
Direct Limits in an Equivariant $K$ Theory Defined by Proper Cocycles
}

\author{
PETER HASKELL
}

Having observed the important role played in a variety of settings by the $K$ theory of the reduced group $C^{*}$ algebras of infinite discrete groups, Baum and Connes [2] considered the $K$ theory of the reduced crossed product $C^{*}$ algebras arising from smooth actions of Lie groups on smooth manifolds. The fundamental insights of [2] are that for a group $G$ and a manifold $X$ as above: one can use as cocycles manifolds on which $G$ acts smoothly and properly to define groups $K^{*}(G, X)$; it is reasonable to conjecture that $K^{*}(G, X)$ is isomorphic to $K_{*}\left(C^{*}\left(G, C_{0}(X)\right)\right)$ via an index map; and if this conjecture is true, then so are many other interesting conjectures. (Because all crossed products are reduced in this paper, the subscript $r$ will be omitted from the notation.)

The definition of $K^{*}(G, X)$ using vector bundles with finite-dimensional fibers that was suggested in [2] was replaced in [3] by a slightly different definition. Because it may be that neither [2] nor [3] is available to the reader and because both are sketchy in their treatment of details, the first section of the present paper is a full discussion of the definition given in [3]. A remark at the end of the paper discusses the role of finite-dimensional vector bundles.

Our main result is a theorem concerning the behavior of $K^{*}(G, X)$ under certain direct limits, of the type $G=\lim _{i} G_{i}$, that is analogous to a theorem about $K_{*}\left(C^{*}\left(G, C_{0}(X)\right)\right)$. Not only does the analogy between these theorems support the Baum-Connes conjecture, but it can be used to prove the conjecture for any Lie group which is the direct limit of open and closed compact subgroups (e.g., a discrete group in which each finitely generated subgroup is finite) and for any abelian Lie group. The main theorem is stated and proved in Section 2, where the above-mentioned cases of the conjecture are proved as corollaries. The proof of the main theorem is interesting in that it illustrates the role that the classifying spaces introduced in [12] and [13] play in the $K$ theory of transformation group $C^{*}$ algebras.

It is a pleasure to thank Paul Baum for suggesting the subject of this paper to me and for many discussions, both broad and narrow, on its subject

Received October 16, 1987. Revision received May 14, 1988.

This material is based upon work supported by the National Science Foundation under Grant Nos. MCS-8301441 and DMS-8501513 and by the Mathematical Sciences Research Institute. Michigan Math. J. 36 (1989). 
matter. I am also grateful to Larry Brown, Jerry Kaminker, Jamie Mingo, Chris Phillips, and Jonathan Rosenberg for their helpful comments.

\section{1. $K^{*}(G, X)$}

Henceforth all groups are Lie groups, and all manifolds, maps, and group actions are smooth. Each group and manifold is assumed to be Hausdorff, second countable, and locally compact, but it may have a countably infinite number of connected components. All vector bundles have finite-dimensional fibers. In discussing the $K$ theory of $C^{*}$ algebras we sometimes adopt the notation of $K K$ theory, for example, $K_{0}(A)=K K(\mathbf{C}, A)$ ([4], [10], [11]), because we use the Kasparov product in some constructions.

In this section we define the groups $K^{*}(G, X)$ associated to a $G$-manifold $X$, that is, to a left action of a group $G$ on a manifold $X$. We use $L_{g}$ to denote the diffeomorphism of $X$ effected by $g \in G$.

DEFINITION. The action of a locally compact group $G$ on a locally compact space $Y$ is said to be proper if, under the map $G \times Y \rightarrow Y \times Y$ given by $(g, y) \rightarrow\left(y, L_{g}(y)\right)$, the inverse image of each compact set is compact. In this situation $Y$ is called a proper $G$-space.

In our situation this definition is equivalent to that in [13]. We proceed to define terms and describe constructions we will need.

If $Z$ is a proper $G$-manifold and $X$ is a $G$-manifold, then $Z \times X$ is a proper $G$-manifold [13]. If $V$ is a $G$-vector bundle over the proper $G$-manifold $Z$, then the total space of $V$, which we denote $E(V)$, is a proper $G$-manifold under the naturally defined action. The pullback of a $G$-vector bundle under an equivariant map between $G$-spaces is a $G$-vector bundle. A proper $G$ manifold can be given a $G$-invariant Riemannian metric [13].

A Riemannian metric on a manifold $Y$ enables us to identify in a smooth manner fibers of $T Y$ with the corresponding fibers of $T^{*} Y$. We will call the identification $T_{y} Y \rightarrow T_{y}^{*} Y$ dual $_{y}$. Let $h: Y_{1} \rightarrow Y_{2}$. The differential $(d h)_{y}$ gives maps $T_{y} Y_{1} \rightarrow T_{h(y)} Y_{2}$ for each $y \in Y_{1}$. Dual $h(y){ }^{\circ}(d h)_{y} \circ$ dual $_{y}^{-1}$ gives maps $T_{y}^{*} Y_{1} \rightarrow T_{h(y)}^{*} Y_{2}$, which piece together to define a map $E\left(T^{*} Y_{1}\right) \rightarrow E\left(T^{*} Y_{2}\right)$. A special case is when $h$ is the map $L_{g}: Y \rightarrow Y$ effected by an element $g$ of $G$. Then the above construction defines a map $E\left(T^{*} Y\right) \rightarrow E\left(T^{*} Y\right)$, making $T^{*} Y$ into a $G$-vector bundle and making $E\left(T^{*} Y\right)$ into a $G$-manifold. With this $G$-action on the total spaces of cotangent bundles, the construction above, applied to an equivariant $f: Y_{1} \rightarrow Y_{2}$, gives rise to an equivariant map between the $G$-manifolds $E\left(T^{*} Y_{1}\right)$ and $E\left(T^{*} Y_{2}\right)$.

If we have maps of manifolds $f_{1}: Y_{1} \rightarrow Y_{3}, f_{2}: Y_{2} \rightarrow Y_{3}$, and $h: Y_{1} \rightarrow Y_{2}$ with $f_{2} \circ h=f_{1}$, then for each $y \in Y_{1}$ the vector spaces $\left(f_{1}^{*} T^{*} Y_{3}\right)_{y}$ and $\left(f_{2}^{*} T^{*} Y_{3}\right)_{h(y)}$ are identified with $T_{f_{1}(y)}^{*} Y_{3}$. Thus for each $y \in Y_{1}$ it makes sense to talk about the identity map of vector spaces $\left(f_{1}^{*} T^{*} Y_{3}\right)_{y} \rightarrow\left(f_{2}^{*} T^{*} Y_{3}\right)_{h(y)}$. We can take the direct sum of this map with the map $\operatorname{dual}_{h(y)}{ }^{\circ}(d h)_{y^{\circ}}$ dual $_{y}^{-1}$ of the preceding paragraph, and the resulting maps can be pieced together to give a 
map $\tilde{h}: E\left(T^{*} Y_{1} \oplus f_{1}^{*} T^{*} Y_{3}\right) \rightarrow E\left(T^{*} Y_{2} \oplus f_{2}^{*} T^{*} Y_{3}\right)$. If $Y_{1}, Y_{2}$, and $Y_{3}$ are $G$ manifolds with $Y_{1}$ and $Y_{2}$ proper, and if $f_{1}, f_{2}$, and $h$ are equivariant, then the resulting map $\tilde{h}: E\left(T^{*} Y_{1} \oplus f_{1}^{*} T^{*} Y_{3}\right) \rightarrow E\left(T^{*} Y_{2} \oplus f_{2}^{*} T^{*} Y_{3}\right)$ is an equivariant map of proper $G$-manifolds.

DEFINITION. A map $h: Y_{1} \rightarrow Y_{2}$ is called $K$-oriented if $T Y_{1} \oplus h^{*} T Y_{2}$ has a $\operatorname{spin}^{c}$ structure.

Examples of $K$-oriented maps include an imbedding with $\operatorname{spin}^{c}$ normal bundle and the projection from the Cartesian product of two manifolds to the second factor when the first factor is a $\operatorname{spin}^{c}$ manifold. In each case in this paper, the key idea is that the direct sum of a vector bundle with itself (or, for that matter, with its dual) has a natural complex structure and thus a natural $\operatorname{spin}^{c}$ structure. In the following general discussion we assume that the fiber dimension of $T Y_{1} \oplus h^{*} T Y_{2}$ is even. That will always be the case in our applications.

Associated to a $K$-oriented map $h: Y_{1} \rightarrow Y_{2}$ is an element $h$ ! of

$$
K K\left(C_{0}\left(Y_{1}\right), C_{0}\left(Y_{2}\right)\right)
$$

([7], [8]). We first describe $h$ ! for two cases: the case when $h$ is an imbedding with complex normal bundle and the case when $h$ is the projection for a fiber bundle in which the fibers are almost complex manifolds and in which the structure group preserves the almost complex structure.

When $h: Y_{1} \rightarrow Y_{2}$ is an imbedding with complex normal bundle (and the image of the imbedding does not "wrap arbitrarily close to itself"), a tubular neighborhood of the image of $h$ can be given the structure of a complex vector bundle $p: F \rightarrow Y_{1}$. "The" Thom class associated to $F$, represented by the module of sections of $p^{*} \Lambda^{*} F$ and the operator exterior product plus its adjoint [1], gives an element of $K K\left(C_{0}\left(Y_{1}\right), C_{0}\left(Y_{2}\right)\right)$. The action of an element $a$ of $C_{0}\left(Y_{1}\right)$ on the module is by multiplication by $a \circ p$. The action of an element $b$ of $C_{0}\left(Y_{2}\right)$ is by multiplication by the restriction of $b$ to the tubular neighborhood.

When $h: Y_{1} \rightarrow Y_{2}$ is a projection as described above, $h$ ! is constructed as follows. Give $Y_{1}$ a Riemannian metric. For each $y \in Y_{2}$ we can form the Hilbert space $H_{y}$ of $L^{2}$ forms of type $(0, *)$ on $h^{-1}(y)$. Let $D_{y}=\bar{\partial}+\bar{\partial}^{*}$ on this Hilbert space, where $\bar{\partial}^{*}$ is the formal adjoint of $\bar{\partial}$. [For concreteness, initially define the operator $\bar{\partial}+\bar{\partial}^{*}$ to have domain equal to the set of smooth compactly supported $(0, *)$-forms. Let $D_{y}$ be the closure of this operator. The class of the $K K$ cycle constructed from $D_{y}$ is not changed if we choose a different "natural" domain for $D_{y}$.] The operator $D_{y} \circ\left(1+D_{y}^{*} D_{y}\right)^{-1 / 2}$ is a bounded operator on the Hilbert space $H_{y}$. These Hilbert spaces patch together to form a bundle of Hilbert spaces over $Y_{2}$. The element $h$ ! of $K K\left(C_{0}\left(Y_{1}\right), C_{0}\left(Y_{2}\right)\right)$ has as its module the set of continuous sections, vanishing at infinity, of this bundle. Its operator $T$ is defined by $(T \sigma)(y)=$ $D_{y^{\circ}}\left(1+D_{y}^{*} D_{y}\right)^{-1 / 2}(\sigma(y)) . C_{0}\left(Y_{1}\right)$ and $C_{0}\left(Y_{2}\right)$ act (respectively) by pointwise multiplication and by pointwise multiplication by the composition with $h$. 
The construction of $h$ ! for such a projection $h$ makes no use of a metric on $Y_{2}$.

With a few more observations we are prepared to use the ! construction in this paper. One observation is that every map $h$ for which we use $h$ ! can be factored into the composition of an imbedding and a projection of the types described above. For $K$-oriented maps $h_{1}: Y_{1} \rightarrow Y_{2}$ and $h_{2}: Y_{2} \rightarrow Y_{3}$, $h_{2} \circ h_{1}$ is $K$-oriented and $\left(h_{2} \circ h_{1}\right) !=h_{1} ! \otimes_{C_{0}\left(Y_{2}\right)} h_{2} !$ [8].

The significance of $h$ ! is that it realizes a map of $K$ theory via the Kasparov product. Let $h: Y_{1} \rightarrow Y_{2}$. For $\alpha \in K K\left(\mathbf{C}, C_{0}\left(Y_{1}\right)\right)$,

$$
\alpha \otimes C_{C_{0}\left(Y_{1}\right)} h ! \in K K\left(\mathrm{C}, C_{0}\left(Y_{2}\right)\right) \text {. }
$$

Replacing $C_{0}\left(Y_{i}\right)$ by $C_{0}\left(Y_{i}\right) \otimes C_{0}(\mathbf{R})$, we get an analogous map $K^{1}\left(Y_{1}\right) \rightarrow$ $K^{1}\left(Y_{2}\right)$.

The second observation is that the above discussion goes over to equivariant $K K$ theory as follows. Assume $h: Y_{1} \rightarrow Y_{2}$ is a $K$-oriented equivariant map between $G$-spaces. Assume further that the metrics, where needed, and the (almost) complex structures (or, in the more general setting of [7] and [8], the $\operatorname{spin}^{c}$ structures) are $G$-invariant. Then the constructions of $h$ ! give operators that commute with the natural actions of $G$ on their modules, and $h ! \in K K_{G}\left(C_{0}\left(Y_{1}\right), C_{0}\left(Y_{2}\right)\right)$. (See [10] for the definition of $K K_{G}$.) The properties of ! (e.g., $\left(h_{2} \circ h_{1}\right) !=h_{1} ! \otimes_{C_{0}\left(Y_{2}\right)} h_{2}$ !) hold in $K K_{G}$. The proof of this statement follows from the observation that the connection approach to Kasparov products used in [8] extends to $K K_{G}$ ([10], see also [9]). When the $G$ actions are proper, all metrics used in the constructions can and should be chosen to be $G$-invariant. The choices made (e.g., the metrics) affect the cycles but not their classes in $K K_{G}$.

Finally, we need to describe how to use ! in conjunction with $j_{G}$. For $h ! \in$ $K K_{G}\left(C_{0}\left(Y_{1}\right), C_{0}\left(Y_{2}\right)\right), j_{G}(h !) \in K K\left(C^{*}\left(G, C_{0}\left(Y_{1}\right)\right), C^{*}\left(G, C_{0}\left(Y_{2}\right)\right)\right)$. (This $j_{G}$ is analogous to the $j_{G}$ of $[10, \S 6]$, the difference being that we complete in the reduced crossed product $C^{*}$ algebra norm.) We have

$$
j_{G}\left(\left(h_{2} \circ h_{1}\right) !\right)=j_{G}\left(h_{1} !\right) \otimes_{C^{*}\left(G, C_{0}\left(Y_{2}\right)\right)} j_{G}\left(h_{2} !\right) \text {. }
$$

That is, ! followed by $j_{G}$ intertwines composition with the appropriate Kasparov product. Also, $j_{G}(h !)$ realizes a map between crossed product $C^{*}$ algebras via Kasparov product in the following manner. For $\alpha \in K K\left(\mathbf{C}, C^{*}\left(G, C_{0}\left(Y_{1}\right)\right)\right)$ ard (resp.) $K K\left(\mathbf{C}, C^{*}\left(G, C_{0}\left(Y_{1}\right)\right) \otimes C_{0}(\mathbf{R})\right)$,

$$
\alpha \otimes_{C^{*}\left(G, C_{0}\left(Y_{1}\right)\right)} j_{G}(h !) \in K K\left(\mathbf{C}, C^{*}\left(G, C_{0}\left(Y_{2}\right)\right)\right)
$$

and (resp.)

$$
K K\left(\mathbf{C}, C^{*}\left(G, C_{0}\left(Y_{2}\right)\right) \otimes C_{0}(\mathbf{R})\right) .
$$

(Of course, $K K\left(\mathbf{C}, A \otimes C_{0}(\mathbf{R})\right.$ ) $=K_{1}(A)$.)

We can now define the groups $K^{*}(G, X)$ associated to a $G$-manifold $X$. For $i=0$ or 1 a $K^{i}$ cocycle for $(G, X)$ is a triple $(Z, \xi, f)$ such that: 
(1) $Z$ is a proper $G$-manifold;

(2) $f: Z \rightarrow X$ is an equivariant map; and

(3) $\xi \in K_{i}\left(C^{*}\left(G, C_{0}\left(E\left(T^{*} Z \otimes f^{*} T^{*} X\right)\right)\right)\right)$.

[It can be instructive to think of $\xi$ as a sort of coefficient, carrying both multiplicity and orientation information for $Z$. A trivial $\xi$ carries only multiplicity information equal to the dimension of its fibers, but a nontrivial $\xi$ puts some extra structure on its cycle. An analogous thing happens in bordism theory where a cycle is described not by a manifold and a map alone but requires the additional information given by the assignment to the manifold of a structure (orientation, stably almost complex structure, etc.) appropriate to the bordism theory.]

We say that there is an elementary equivalence of $K^{i}$ cocycles $\left(Z_{1}, \xi_{1}, f_{1}\right) \sim$ $\left(Z_{2}, \xi_{2}, f_{2}\right)$ if there is an equivariant map $h: Z_{1} \rightarrow Z_{2}$ such that:

(1) $f_{1}=f_{2} \circ h$; and

(2) for the map $\tilde{h}: E\left(T^{*} Z_{1} \oplus f_{1}^{*} T^{*} X\right) \rightarrow E\left(T^{*} Z_{2} \oplus f_{2}^{*} T^{*} X\right)$ formed from $h, \xi_{1} \otimes_{C^{*}\left(G, C_{0}\left(E\left(T^{*} Z_{1} \oplus f_{1}^{*} T^{*} X\right)\right)\right)} j_{G}(\tilde{h} !)=\xi_{2}$.

(The map $\tilde{h}$ is $K$-orientable because of the natural complex structure of the direct sum of a vector bundle with itself or with its dual.)

DEFINITION. $K^{i}(G, X)$ is defined to be $\left\{K^{i}\right.$ cocycles for $\left.(G, X)\right\} /$ the equivalence relation generated by elementary equivalences.

The disjoint union of $K^{*}$ cocycles realizes addition in $K^{*}(G, X)$. Also, $(Z,-\xi, f)=-(Z, \xi, f)$.

One can define a map

$$
\mu: K^{*}(G, X) \rightarrow K_{*}\left(C^{*}\left(G, C_{0}(X)\right)\right)
$$

as follows. Let $(Z, \xi, f)$ be a $K^{*}$ cocycle for $(G, X)$. Let $\pi$ be the projection $E\left(T^{*} Z \oplus f^{*} T^{*} X\right) \rightarrow Z$. Then $\mu((Z, \xi, f))$ is

$$
\xi \otimes_{C^{*}\left(G, C_{0}\left(E\left(T^{*} Z+f^{*} T^{*} X\right)\right)\right)} j_{G}((f \circ \pi) !) .
$$

Because $j_{G}(\cdot !)$ intertwines composition with the Kasparov product and because the Kasparov product is associative, $\mu$ is well-defined on $K^{*}(G, X)$. The Baum-Connes conjecture is that $\mu$ is always an isomorphism.

We finish this section with two observations. First, the conjecture that $\mu: K^{*}(G, X) \rightarrow K_{*}\left(C^{*}\left(G, C_{0}(X)\right)\right)$ is an isomorphism is true when $X$ is a proper $G$-manifold. The argument has two ingredients. One is the Thom isomorphism $K_{*}\left(C^{*}\left(G, C_{0}\left(E\left(T^{*} X \oplus T^{*} X\right)\right)\right)\right)=K_{*}\left(C^{*}\left(G, C_{0}(X)\right)\right)$ of [14]. The other is that cycles of the form $\left(X, \xi\right.$, id) with $\xi \in K_{*}\left(C^{*}\left(G, C_{0}\left(T^{*} X \oplus T^{*} X\right)\right)\right)$ are "final" for $K^{*}(G, X)$. When $G$ is compact, the Green-Julg theorem says that the groups in the domain and range of $\mu$ are isomorphic to the equivariant $K$ theory groups defined by Segal [4]. 
Second, in using ! (say, to define the equivalence relation or to define $\mu$ ) we can restrict ! to maps that are imbeddings with complex normal bundles or the projections of fiber bundles whose fibers have almost complex structures preserved by the structure group. The reason is that $h: Z \rightarrow Y$ can be factored by (id, $h): Z \rightarrow Z \times Y$ and $p_{Y}: Z \times Y \rightarrow Y$. Our constructions require us to apply ! to maps constructed from this imbedding and projection and defined on the total spaces of vector bundles. These maps will be of the kind described in the first sentence of this paragraph.

\section{Direct Limits}

Throughout this section we consider the following situation. Let a Lie group $G$ be equal to the direct limit of Lie subgroups $G_{i}$, with the indices $i$ elements of the positive integers with their usual ordering. Assume further that each $G_{i}$ is an open and closed subgroup of $G$ and that $i_{1}<i_{2}$ implies $G_{i_{1}} \subset$ $G_{i_{2}}$. Some examples appear in the corollaries in this section. An action of $G$ on a space restricts to an action of each $G_{i}$ on the space.

In this section we prove that in this situation $\lim _{i} K^{*}\left(G_{i}, X\right)$ makes sense and is equal to $K^{*}(G, X)$. The significance of this theorem is that there is an analogous result in the $K$ theory of $C^{*}$ algebras: $K_{*}\left(C^{*}\left(G, C_{0}(X)\right)\right)=$ $\lim _{i} K_{*}\left(C^{*}\left(G_{i}, C_{0}(X)\right)\right)$. It follows that the conjectured isomorphism of $\overrightarrow{K^{*}}(G, X)$ and $K_{*}\left(C^{*}\left(G, C_{0}(X)\right)\right)$ reduces to analogous isomorphisms involving the $G_{i}$, which we check in a couple of cases.

We attend first to the well-known $C^{*}$ algebraic results. Direct limits of $C^{*}$ algebras will always be $C^{*}$ direct limits.

LEMMA. Let $A=\lim _{\rightarrow} A_{i}$ be a direct limit of $C^{*}$ algebras. Then $\lim _{\rightarrow} K_{*}\left(A_{i}\right) \cong$ $K_{*}(A)$.

Proof. Considered well-known for years, this lemma receives an explicit proof in a more general setting in [17].

LEMMA. Let $G=\lim _{i} G_{i}$, under the assumptions at the beginning of this section. Suppose $G \overrightarrow{a c t s}$ smoothly on a manifold $X$. Then, for $i, j, k \in \mathbf{Z}_{+}$with $i<j$, there are maps $C^{*}\left(G_{i}, C_{0}(X)\right) \rightarrow C^{*}\left(G_{j}, C^{*}(X)\right)$ and $C^{*}\left(G_{k}, C_{0}(X)\right) \rightarrow$ $C^{*}\left(G, C_{0}(X)\right)$ which realize an isomorphism

$$
\lim _{\vec{i}} C^{*}\left(G_{i}, C_{0}(X)\right)=C^{*}\left(G, C_{0}(X)\right) .
$$

Proof. Letting $H_{1} \subset H_{2}$ denote either $G_{i} \subset G_{j}$ or $G_{k} \subset G$, define

$$
C_{c}\left(H_{1}, C_{0}(X)\right) \rightarrow C_{c}\left(H_{2}, C_{0}(X)\right)
$$

to be extension by zero. Because $H_{2} / H_{1}$ is discrete, these maps extend to $C^{*}$ algebra homomorphisms with the desired properties.

We now consider the behavior of $K^{*}(G, X)$ under such direct limits. Let $H_{1}$ and $\mathrm{H}_{2}$ have the meaning given them in the proof of the preceding lemma. 
On the level of cocycles, we define the map ind: $K^{*}\left(H_{1}, X\right) \rightarrow K^{*}\left(H_{2}, X\right)$ by sending $(Z, \xi, f)$ to $\left(H_{2} \times_{H_{1}} Z, \xi^{\prime}, f^{\prime}\right)$. It is easily checked that when $Z$ is a proper $H_{1}$-manifold $H_{2} \times{ }_{H_{1}} Z$, which is defined to be $\left(H_{2} \times Z\right) /((g h, z) \sim$ $(g, h z)$ for $g \in H_{2}$ and $\left.h \in H_{1}\right)$, is a proper $H_{2}$-manifold under the action $g_{1}[(g, z)]=\left[\left(g_{1} g, z\right)\right]$. The map $f^{\prime}$ is determined by the requirement that it equivariantly extend $f$. Having observed that $H_{2} / H_{1}$ discrete implies that $E\left(T^{*}\left(H_{2} \times_{H_{1}} Z\right) \oplus f^{*} T^{*} X\right)=H_{2} \times_{H_{1}} E\left(T^{*} Z \oplus f^{*} T^{*} X\right)$, we define $\xi^{\prime}$ to be $i(\xi)$, where $i$ is the induction isomorphism

$$
\begin{aligned}
& K_{*}\left(C^{*}\left(H_{1}, C_{0}\left(E\left(T^{*} Z \oplus f^{*} T^{*} X\right)\right)\right)\right) \\
& \quad \rightarrow K_{*}\left(C^{*}\left(H_{2}, C_{0}\left(H_{2} \times_{H_{1}}\left(E\left(T^{*} Z \oplus f^{*} T^{*} X\right)\right)\right)\right)\right)
\end{aligned}
$$

arising from the strong Morita equivalence of the $C^{*}$ algebras [16]. By [10], $i$ can be realized by taking the Kasparov product on the right over the original $C^{*}$ algebra with the equivalence bimodule of [16].

It remains to be checked that ind, as constructed on cocycles, describes a well-defined map $K^{*}\left(H_{1}, X\right) \rightarrow K^{*}\left(H_{2}, X\right)$. We also need to know that these maps determine a direct system $K^{*}\left(G_{i}, X\right)$ and that

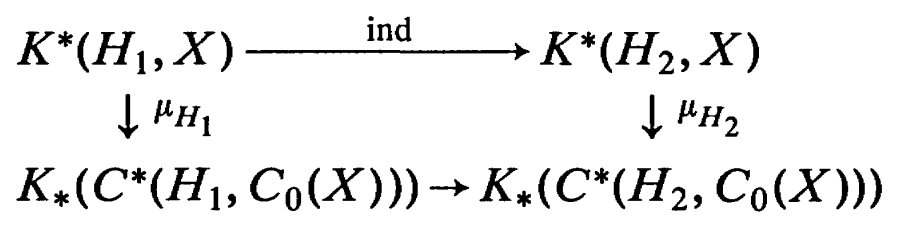

commutes. (The lower horizontal map in the diagram is the one coming from the $C^{*}$ algebra map described in the proof of the preceding lemma.) We leave the verification of these claims to the reader but offer two suggestions about the proofs. First, these statements are checked by direct computation of the $K K$ elements, the products with which realize the various maps. Second, it is easier to factor maps through imbeddings and submersions when doing these computations.

THEOREM. $\operatorname{Lim}_{\rightarrow} K^{*}\left(G_{i}, X\right) \cong K^{*}(G, X)$.

Proof. We start by establishing surjectivity of the map from left to right. By [14], we need consider only cycles $(Z, \xi, f)$ for which $\xi$ has $G$-compact support. (In the definition of $K_{*}\left(C^{*}\left(G, C_{0}(W)\right)\right)$ established in [14] for proper actions of $G$ on $W$, cycles are represented by Hilbert vector bundles with morphisms between them. The support contains the points over which the morphism is not invertible. For our purposes it is sufficient to know that all of the $K$-theoretic information carried by $\xi$ is carried by the restriction of $\xi$ to a neighborhood of its support.) Because $E\left(T^{*} Z \oplus f^{*} T^{*} X\right)$ is locally compact, we can find a compact neighborhood of support $(\xi) / G$. Let $Z^{\prime}$ be the pre-image in $E\left(T^{*} Z \oplus f^{*} T^{*} X\right)$ of this neighborhood, and let $Y$ be the interior of $Z^{\prime}$. Let $j$ be the composition of $f$ with the projection from $Y$ to $Z$. Let $t$ be the Thom isomorphism

$$
K_{*}\left(C^{*}\left(G, C_{0}(Y)\right)\right) \rightarrow K_{*}\left(C^{*}\left(G, C_{0}\left(E\left(T^{*} Y \oplus j^{*} T^{*} X\right)\right)\right)\right) .
$$


We now consider the cocycle $(Y, t(\xi), j)$, which represents the same class in $K^{*}(G, X)$ as $(Z, \xi, f)$.

Because $Y$ is contained in the $G$-compact set $Z^{\prime}$, we can draw two conclusions. First, we can argue as in [5] that $Y$ contains only finitely many orbit types. Thus the theory of classifying spaces for proper $G$-actions ([12], [13]) applies. Second, the image of $Y / G$ under the classifying map is contained in a compact subset of the appropriate classifying space. This means that $Y$ is equivalent to an induced $G$-space $G \times_{G^{\prime}} Y^{\prime}$, where $G^{\prime}$ is one of the $G_{i}$. Our discussion of the map ind completes the proof of this theorem once we outline the proof of the claim that $Y$ is $G \times_{G^{\prime}} Y^{\prime}$. We now do this.

Let $X_{1}, \ldots, X_{n}$ be proper $G$-spaces and let $I$ be the unit interval. The reduced join of the $\left\{X_{i}\right\}$ is formed as follows. Take $\left\{\left(\left(x_{1}, t_{1}\right), \ldots,\left(x_{n}, t_{n}\right)\right): \Sigma t_{i}=1\right\}$. Form the quotient by the equivalence relation $\left(\left(x_{1}, t_{1}\right), \ldots,\left(x_{n}, t_{n}\right)\right) \sim$ $\left(\left(y_{1}, s_{1}\right), \ldots,\left(y_{n}, s_{n}\right)\right)$ if and only if (a) $t_{i}=s_{i}$ for all $i$ and (b) $t_{i} \neq 0$ implies $x_{i}=y_{i}$ for all $i$. The reduced join is the subset of this quotient defined as follows. For $p=\left(\left(x_{1}, t_{1}\right), \ldots,\left(x_{n}, t_{n}\right)\right)$ let $K_{i, p}$ be the isotropy group of $x_{i}$ if $t_{i} \neq 0$ and $G$ if $t_{i}=0$. Then $p$ is in the reduced join if and only if there is a $K_{i, p}$ contained in all of the $K_{j, p}$. The action of $G$ on the reduced join is given by $g\left(\left(x_{1}, t_{1}\right), \ldots,\left(x_{n}, t_{n}\right)\right)=\left(\left(g x_{1}, t_{1}\right), \ldots,\left(g x_{n}, t_{n}\right)\right)$.

Let $\left\{H_{r}\right\}$ be a set of representatives of the conjugacy classes of isotropy groups appearing in the $Y$ introduced in the second paragraph of this proof. Palais' universal space $U$ is a reduced join of copies of the $G / H_{r}$, where each $G / H_{r}$ may appear more than once. $U$ is a proper $G$-space, and $U / G$ is the classifying space governing the action of $G$ on $Y$.

For each $G_{i}$ containing all of the $H_{r}, U / G$ contains a subspace that can be identified with the classifying space for certain proper actions of $G_{i}$. This subspace is described as $\left\{\left[\left(\left(x_{1}, t_{1}\right), \ldots,\left(x_{n}, t_{n}\right)\right)\right] \in U / G\right.$ : for all $u, v$ with $t_{u} \neq$ 0 and $t_{v} \neq 0, x_{v}^{-1} x_{u} \subset G_{i}$. Let $P$ be the natural projection

$$
U / G \rightarrow\left\{\left(t_{1}, \ldots, t_{n}\right): \Sigma t_{i}=1\right\} .
$$

Observe that there is a natural simplicial decomposition of the image of this projection. The "fibers" of $P$ are constant over the interior of each simplex in this decomposition. Also, if simplex $\sigma_{1}$ is contained in the boundary of simplex $\sigma_{2}$ and if the fibers of $P$ over the interior of $\sigma_{2}$ are not empty, then they are "at least as big as" the fibers over interior points in $\sigma_{1}$. (To understand the phrase in quotes, regard it as a necessary condition for $U / G$ to be Hausdorff.)

It follows that if there exists an infinite collection of proper containments, classifying space for $G_{i}$ properly contained in classifying space for $G_{i+1}$, then this can be observed on some fiber of $P$. This fiber is then noncompact, and the reasoning of the preceding paragraph shows that each compact subset of $U / G$ is contained in one of the classifying spaces for $G_{i}$ actions. Of course, if there are only finitely many proper containments of classifying spaces then the claim is true as well. This completes the proof of surjectivity. 
The proof of injectivity is much the same. It suffices to check that, if two cocycles for $K^{*}(G, X)$ are related by an elementary equivalence, then they (are cocycles for and) are related by an elementary equivalence for some $K^{*}\left(G_{i}, X\right)$. Suppose $h: Z_{1} \rightarrow Z_{2}$ realizes an elementary equivalence $\left(Z_{1}, \xi_{1}, f_{1}\right) \sim\left(Z_{2}, \xi_{2}, f_{2}\right)$. (Assume $*=0$; if $*=1$, the same arguments work when we replace the total spaces of the relevant vector bundles by the Cartesian products of these spaces with $\mathbf{R}$.) By [14], the equivalence relation in $K_{0}\left(C^{*}\left(G, C_{0}\left(E\left(T^{*} Z_{2} \oplus f^{*} T^{*} X\right)\right)\right)\right)$ is generated by the addition of trivial virtual Hilbert vector bundles and by homotopy. Because there is no difficulty in working addition of trivial bundles into our argument, we focus on homotopy. The homotopy consists of a pair of Hilbert vector bundles over $E\left(T^{*} Z_{2} \oplus f^{*} T^{*} X\right) \times[0,1]$ with a compactly supported morphism between them. (See the definition of support in the first paragraph of this proof.) As in the proof of surjectivity, the compact support is used to deduce that the homotopy is induced from a homotopy at the level of one of the $G_{i}$. This completes the proof of the theorem.

COROLLARY. The Baum-Connes conjecture is true if each $G_{i}$ is compact.

Proof. Because $G_{i}$ is compact, every smooth $G_{i}$-action is proper and so the conjecture is true at each stage of the direct limit.

COROLLARY. The Baum-Connes conjecture is true for abelian Lie groups.

Proof. For some choice of finite $m$ and $n, G=\mathbf{T}^{m} \oplus \mathbf{R}^{n} \oplus$ a countably generated discrete abelian group. Let $G_{i}$ be the subgroup $\mathbf{T}^{m} \oplus \mathbf{R}^{n} \oplus$ discrete group generated by the first $i$ generators of the discrete group. To proceed as in the proof of the preceding corollary, we need only prove: (a) if the Baum-Connes conjecture is true for $H$ then it is true for $H \oplus \mathbf{R}$; and (b) if the conjecture is true for $H$ then it is true for $H \oplus \mathbf{Z}$.

To prove (a), observe that there is an equivalence of categories between the cocycles for $K^{i}(H \oplus \mathbf{R}, X)$ and the cocycles for $K^{i+1}(H, X)$. This equivalence arises because $\mathbf{R}$ is a contractible torsion-free group. This equivalence takes a cocycle $(Z, \xi, f)$ for $K^{i+1}(H, X)$ to $\left(Z \times \mathbf{R}, s(\xi), f_{1}\right)$. The action of $H \oplus \mathbf{R}$ on $Z \times \mathbf{R}$ is defined by letting $H$ act on $Z$ and letting $\mathbf{R}$ act by addition on $\mathbf{R}$. The map $f_{1}$ is determined by requiring that it equivariantly extend $f$. The map $s$ is the inverse of the composition

$$
\begin{aligned}
K^{i}\left(C^{*}\right. & \left.\left(H \oplus \mathbf{R}, C_{0}\left(E\left(T^{*}(Z \times \mathbf{R}) \oplus f_{1}^{*} T^{*} X\right)\right)\right)\right) \\
& \rightarrow K^{i}\left(C^{*}\left(H, C_{0}\left(E\left(T^{*} Z \oplus f^{*} T^{*} X\right) \times \mathbf{R}\right)\right)\right) \\
& \rightarrow K^{i+1}\left(C^{*}\left(H, C_{0}\left(E\left(T^{*} Z \oplus f^{*} T^{*} X\right)\right)\right)\right),
\end{aligned}
$$

where the first map is the isomorphism of [14, Thm. 8.1] and the second follows from definition, Bott periodicity, and the triviality of the action of $H$ on $\mathbf{R}$. To finish the proof of (a) observe that $\mu$ intertwines the isomorphism induced by this equivalence of categories with the isomorphism established in [6] for the $K$ theory of $C^{*}$ algebras. 
To prove (b), let $\mathbf{Z}$ act on $\mathbf{R}$ by addition and let $\mathbf{Z}$ act on $\mathbf{R} \times X$ diagonally. There is an equivalence of categories between the cocycles for $K^{i}(\mathbf{Z} \oplus H, X)$ and those for $K^{i+1}(H,(\mathbf{R} \times X) / Z)$. This equivalence takes a cocycle $(Z, \xi, f)$ for $K^{i}(\mathrm{Z} \oplus H, X)$ to $\left(Z / Z, \xi_{1}, f_{1}\right)$, which is constructed as follows. Because $\mathbf{Z}$ is torsion-free, a proper action of $\mathbf{Z}$ on $Z$ is a principal $\mathbf{Z}$-bundle. Thus there is an equivariant map $f_{0}: Z \rightarrow \mathbf{R} \times X$ which drops to the quotients to give $f_{1}: Z / Z \rightarrow(\mathbf{R} \times X) / Z$. $\xi_{1}$ is constructed from $\xi$ by a map analogous to the inverse of $s$ in the preceding paragraph. The equivalence of categories gives the first isomorphism in the following string of isomorphisms:

$$
\begin{aligned}
K^{i}(\mathbf{Z} \oplus H, X) & \rightarrow K^{i+1}(H,(\mathbf{R} \times X) / \mathbf{Z}) \rightarrow K_{i+1}\left(C^{*}\left(H, C_{0}((\mathbf{R} \times X) / \mathbf{Z})\right)\right) \\
& \rightarrow K_{i}\left(C^{*}\left(H \oplus \mathbf{R}, C_{0}((\mathbf{R} \times X) / \mathbf{Z})\right)\right) \\
& \rightarrow K_{i}\left(C^{*}\left(H, C^{*}\left(\mathbf{Z}, C_{0}(X)\right)\right)\right) \rightarrow K_{i}\left(C^{*}\left(\mathbf{Z} \oplus H, C_{0}(X)\right)\right) .
\end{aligned}
$$

The other isomorphisms arise by assumption, [6], strong Morita equivalence, and the commuting of the $\mathbf{Z}$ and $H$ actions. A check that the composition agrees with $\mu$ completes the proof of this corollary.

REMARK. The original paper [2] suggested that $K^{*}(G, X)$ could be defined with proper cocycles carrying equivariant finite-dimensional vector bundles. An example in [14] shows that for $G$ acting properly on $X, K^{*}\left(C^{*}\left(G, C_{0}(X)\right)\right)$ cannot always be defined using only finite-dimensional vector bundles on $X$. This example does not imply that $K^{*}(G, X)$ cannot always be defined using only finite-dimensional vector bundles. However, it does imply that if one wants to define $K^{*}(G, X)$ using only finite-dimensional vector bundles, one loses the argument that says that $K^{*}(G, X)$ is isomorphic to $K_{*}\left(C^{*}\left(G, C_{0}(X)\right)\right)$ when $G$ acts properly on $X$ because $X$ is a final cocycle in the direct system. This seems too large a price to pay. Phillips [14] shows that much of the geometric character of $K^{*}(G, X)$ can be retained if one accepts vector bundles with infinite-dimensional fibers. In fact, in families' index theorems these arise more naturally than finite-dimensional vector bundles. However, it is sometimes useful to have finite-dimensional vector bundles. Phillips [15] has shown that these are sufficient for some Lie groups $G$. The present paper shows that finite-dimensional vector bundles suffice to define $K^{*}(G, X)$ when $G$ is a direct limit of groups for which finite-dimensional bundles suffice.

\section{References}

1. M. Atiyah, R. Bott, and A. Shapiro, Clifford modules, Topology 3 (1964), 229247.

2. P. Baum and A. Connes, Geometric $K$ theory for Lie groups and foliations, preprint, 1982.

3. $-K$ theory for actions of discrete groups, preprint, 1985.

4. B. Blackadar, K-theory for operator algebras, MSRI Publ. 5, Springer, New York, 1986. 
5. G. Bredon, Introduction to compact transformation groups, Academic Press, New York, 1972.

6. A. Connes, An analogue of the Thom isomorphism for crossed products of a $C^{*}$ algebra by an action of $\mathbf{R}$, Adv. in Math. 39 (1981), 31-55.

7. - A survey of foliations and operator algebras, Proc. Sympos. Pure Math., 38, Amer. Math. Soc., Providence, R.I., 1982.

8. A. Connes and G. Skandalis, The longitudinal index theorem for foliations, Publ. Res. Inst. Math. Sci. 20 (1984), 1139-1183.

9. J. Fox, P. Haskell, and I. Raeburn, Kasparov products, KK equivalence, and proper actions of connected reductive Lie groups, J. Operator Theory, to appear.

10. G. Kasparov, Equivariant $K K$-theory and the Novikov conjecture, Invent. Math. 91 (1988), 147-201.

11. - The operator $K$-functor and extensions of $C^{*}$-algebras, Math. USSRIzv. 16 (1981), 513-572.

12. R. Palais, The classification of G-spaces, Mem. Amer. Math. Soc. 36 (1960).

13. - On the existence of slices for actions of non-compact Lie groups, Ann. of Math. (2) 73 (1961), 295-323.

14. N. C. Phillips, Equivariant K-theory for proper actions, preprint.

15. - Equivariant K-theory for proper actions II: Some cases in which finite dimensional bundles suffice, Index theory of elliptic operators, foliations, and operator algebras (J. Kaminker, K. Millett, C. Schochet, eds.), Con. Math., 70, Amer. Math. Soc., Providence, R.I., 1988.

16. M. Rieffel, Applications of strong Morita equivalence to transformation group $C^{*}$-algebras, Proc. Sympos. Pure Math., 38, Amer. Math. Soc., Providence, R.I., 1982.

17. J. Rosenberg and C. Schochet, The Kunneth and the universal coefficient theorem for Kasparov's generalized K-functor, Duke Math. J. 55 (1987), 431-474.

Mathematics Department

Virginia Tech.

Blacksburg, VA 24061 
